\title{
Reprodutibilidade intraobservador do teste Timed Up and Go para pacientes com DPOC
}

\section{Intrarater reliability of Timed Up and Go Test to patients with COPD}

\author{
Henrique da Conceição Costa ${ }^{1}$ \\ Barbara Silva dos Santos ${ }^{2}$ \\ Natasha Cordeiro dos Santos ${ }^{3}$
}

\section{Taís Santana Barbosa ${ }^{4}$ \\ Aline Gonçalves Miranda 5 (1) Aquiles Assunção Camelier ${ }^{6}$ (1) Fernanda Warken Rosa Camelier ${ }^{7}$ (1)}

1-5Universidade do Estado da Bahia. (Salvador).Bahia, Brasil.costahdc@outlook.com,barbarasilva649@gmail.com, natasha-cordeiro@hotmail.com, taisbarbosa_@outlook.com, alinegoncalves4391@gmail.com ${ }^{6}$ Escola Bahiana de Medicina e Saúde Pública e Universidade do Estado da Bahia. (Salvador). Bahia, Brasil. aquilescamelier@yahoo.com.br ${ }^{7}$ Autora para correspondência. Universidade do Estado da Bahia. (Salvador). Bahia, Brasil. fcamelier@uneb.br

RESUMO | INTRODUÇÃO: O Timed UP and Go Test (TUG) é um importante teste utilizado para a avaliação de desfechos funcionais na DPOC, como mobilidade, equilíbrio dinâmico e risco de queda. No cenário clínico prático, a aplicação repetida do TUG é realizada muitas vezes pelo mesmo observador, no objetivo de avaliar diversas intervenções aplicadas na DPOC. OBJETIVO: Avaliar o grau de reprodutibilidade intraobservador do TUG em indivíduos com DPOC. METODOLOGIA: Estudo descritivo, de corte transversal, realizado com indivíduos com DPOC, atendidos ambulatorialmente na cidade de Salvador-Bahia. Foram verificadas características sociodemográficas e clínicas como a espirometria (VEF/CVF), e tempo de realização de duas práticas no mesmo dia do teste TUG. A reprodutibilidade do TUG foi avaliada pelo Coeficiente de Correlação Intraclasse (CCI), CAAE número 38143214.0.0000.0057. RESULTADOS: Trinta e um pacientes voluntários foram avaliados; destes, 24 (77,4\%) homens, média da idade de 68,6 $\pm 9,8$ anos, com relação $\mathrm{VEF}_{1} /$ CVF pós-broncodilatador de 59,0 $\pm 10,8 \%$. Na análise da reprodutibilidade intraobservador do TUG pelo $\mathrm{CCl}$, obteve-se $a=0,897$ (Cl 95\%: 0,786; 0,950; $p<0,0001)$. CONCLUSÃO: $O$ teste TUG possui excelente reprodutibilidade intraobservador e uma pequena variabilidade quando aplicados duas vezes em pacientes com DPOC, sendo a aplicação para avaliação da mobilidade, na prática do cuidado em saúde considerada factível.

PALAVRAS-CHAVE: Doença Pulmonar Obstrutiva Crônica. Reprodutibilidade dos Testes. Assistência Ambulatorial. Fisioterapia. Confiabilidade dos dados.
ABSTRACT | INTRODUCTION: The Timed UP and Go Test (TUG) is an important test used to assess functional outcomes in COPD, such as mobility, dynamic balance, and risk of falling. In clinical practice, the repeated application of the TUG is performed several times by the same observer to evaluate different interventions applied in COPD. OBJECTIVE: To assess the degree of intraobserver reproducibility of TUG in individuals with COPD. METHODOLOGY: Descriptive, cross-sectional study carried out with individuals with COPD treated in an outpatient clinic in the city of SalvadorBahia. Sociodemographic and clinical characteristics such as spirometry $\left(\mathrm{FEV}_{1} / \mathrm{FVC}\right)$ were verified, as well as two practices of the TUG Test on the same day. The reproducibility of TUG was evaluated fur Intraclass Correlation Coefficient (ICC). CAAE number 38143214.0.0000.0057. RESULTS: Thirty-one volunteer patients were evaluated, of which $24(77.4 \%)$ men, mean age $68.6 \pm 9.8$ years, with post-bronchodilator $\mathrm{FEV}^{1} / / \mathrm{FVC}$ ratio 59.0 $\pm 10.8 \%$. In the analysis of intraobserver reproducibility of the TUG by the ICC, $a=0.897$ (CI 95\%: 0.786; 0.950; $p<0.0001$ ) was obtained. CONCLUSION: The TUG test has excellent intraobserver reproducibility and a small variability when applied twice in patients with COPD, being the application for mobility assessment, in care practice, considered viable.

KEYWORDS: Chronic obstructive pulmonary disease. Test reproducibility. Outpatient Assistance. Physiotherapy. Data reliability. 


\section{Introdução}

A Doença Pulmonar Obstrutiva Crônica (DPOC) é uma enfermidade prevenível e tratável caracterizada pela persistência de sintomas respiratórios e pela limitação ao fluxo de ar, determinadas por anormalidades alveolares e de vias aéreas geralmente causadas pela exposição significante às partículas nocivas ou gases. ${ }^{1}$ Além dessas repercussões, pessoas com DPOC apresentam impactos extrapulmonares como sarcopenia e dentre outros, o que pode contribuir para a intolerância ao exercício1; e quando comparados a indivíduos saudáveis, pacientes com DPOC são menos ativos. ${ }^{2}$ As consequências da redução de força e resistência da musculatura esquelética aumentada pela inatividade do indivíduo podem contribuir para a redução do desempenho na realização de testes funcionais. $\frac{3}{}$

Na prática fisioterapêutica, especificamente na avaliação e assistência de pessoas com pneumopatias crônicas, o que inclui a DPOC, a utilização de testes funcionais acessíveis, eficazes e de baixo custo é cada vez mais frequente, sendo o teste de sentar e levantar, teste de caminhada de seis minutos e o Timed Up and Go Test (TUG) modalidades de avaliação funcional com aplicabilidade reconhecida., $\mathrm{A}$ importância desses métodos se dá principalmente pela necessidade de avaliação dos pacientes, que tem por finalidade diagnosticar funcionalmente e auxiliar na decisão das condutas para o adequado manejo desses indivíduos..

O TUG é considerado uma ferramenta possível de identificar diferentes desfechos em pessoas com DPOC, sendo aplicado na avaliação da mobilidade funcional, equilíbrio estático e dinâmico, capacidade de andar, giro, sentar e levantar, e pode predizer risco de quedas 6 e de sarcopenia em idosos. ${ }^{?}$ O teste consiste no ato de levantar de uma cadeira, andar por três metros, voltar e sentar-se novamente. Idosos com desempenho maior que 13,5 segundos apresentam maior risco de quedas ${ }^{8}$, porém o valor de referência para o teste na população específica de indivíduos com DPOC é de um tempo superior a 11,2 segundos para detectar alterações de mobilidade. ${ }^{4}$

Uma revisão publicada por Holland et al. ${ }^{2}$ identificou que o teste TUG apresenta uma alta confiabilidade teste-reteste em pacientes com DPOC, achado esse comum em pacientes com AVC ${ }^{10}$ (coeficiente de correlação intraclasse $[\mathrm{CCl}]>95$ ), e outros dois estudos avaliaram a responsividade do TUG após um programa de reabilitação pulmonar. $\frac{112}{12} \mathrm{Na}$ maioria dos cenários de cuidado em saúde (ambientes clínicos), torna-se necessário que um avaliador humano integre o sistema de avaliação ao ser aplicado um teste, questionário ou uma escala. $\frac{13}{}$ Neste contexto, há a necessidade da avaliação da reprodutibilidade intraobservador para se quantificar a estabilidade ou a variabilidade dos dados registrados pelo mesmo observador durante o ato da mensuração da característica clínica específica. $\frac{13}{3}$

Apesar do TUG ser um método de avaliação válido, o estudo de propriedades de medida ou clinimétricas, que inclui reprodutibilidade intraobservador, é importante para auxiliar o profissional de saúde na escolha do teste e como aplicá-lo e interpretá-lo na prática. ${ }^{6} \mathrm{O}$ objetivo do estudo foi verificar a reprodutibilidade intraobservador do Timed Up and Go Test em indivíduos com Doença Pulmonar Obstrutiva Crônica.

\section{Metodologia}

Tratou-se de estudo descrito, de corte transversal com uma amostra de conveniência, composta por pessoas com DPOC atendidos ambulatorialmente na cidade de Salvador-Bahia. Foram incluídos na pesquisa os pacientes com diagnóstico de DPOC, estáveis de acordo com o documento GOLD (Global Initiative for Chronic Obstructive Lung Disease) ${ }^{1}$, que realizaram espirometria há pelo menos seis meses a contar da data da avaliação. Foram excluídos os que apresentaram dificuldades na compreensão dos testes.

A coleta de dados foi realizada no Laboratório de Fisiologia do Exercício, Departamento de Ciências da Vida II da Universidade do Estado da Bahia (UNEB), Salvador, Bahia. Os indivíduos foram submetidos a uma avaliação em um único dia, e foram verificadas as características sociodemográficas, além da realização do Teste TUG, por duas vezes em sequência no mesmo dia, com intervalo mínimo de um minuto entre cada teste. Os dados de função pulmonar recente foram obtidos por solicitação ao paciente. 
Para a realização do teste TUG, foram utilizados um cronômetro de acionamento manual disponível no smartphone modelo iPhone 6, uma trena, uma cadeira e um cone. Os indivíduos foram orientados a sentarem-se na cadeira sem apoio, levantarem-se, andarem por três metros demarcados no chão até o cone e se sentarem novamente. O tempo de corte utilizado para a avaliação de indivíduos com DPOC foi $>11,2$ segundos. ${ }^{4}$ As variáveis sociodemográficas consideradas foram idade em anos (mediana); sexo (masculino e feminino); cor da pele (branco, mulato, negro, amarelo); Índice de Massa Corpórea $\left(\mathrm{Kg} / \mathrm{m}^{2}\right)$; peso (Kg); altura (metro). Os pacientes foram separados em grupos conforme a classificação de gravidade da limitação ao fluxo de ar conforme determinados no documento GOLD ${ }^{1}$ considerado mediante avaliação de parâmetros como a capacidade vital forçada (CVF), volume expiratório forçado no primeiro segundo $\left(V_{E F}\right)$ e a relação $V_{E F} / C V F$ pós-broncodilatador (pós-BD). As variáveis clínicas também foram consideradas: saturação periférica de oxigênio $\left(\mathrm{SpO}_{2}\right)$; presença de hipertensão; diabetes; status tabágico e medicações em uso.

Os dados foram armazenados e analisados no software SPSS (v.17.0) e foram expressos em medida de tendência central, dispersão e proporções. Para a comparação do desempenho dos dois testes TUG foi utilizado o Teste T pareado. Utilizou-se a ANOVA para avaliar a diferença entre o tempo de desempenho do TUG entre as classes de limitação de fluxo de ar do GOLD 2021. A reprodutibilidade intraobservador do TUG foi avaliada pelo Coeficiente de Correlação Intraclasse $(\mathrm{CCl})$, sendo assumida baixa reprodutibilidade para valores inferiores a 0,4, entre 0,4 e 0,75, como boa reprodutibilidade e os valores acima de 0,75 foram considerados como reprodutibilidade excelente. ${ }^{14}$ Para análise de dispersão entre os dois testes TUG, traçou-se gráficos de Bland-Altman. Um $\mathrm{p}<$ 0,05 foi considerado estatisticamente significante. Os dados do presente estudo integram a pesquisa aprovada pelo Comitê de Ética e Pesquisa da Universidade do Estado da Bahia (UNEB) sob o parecer 882003.

\section{Resultados}

A amostra foi composta com 31 indivíduos com DPOC atendidos em um ambulatório de referência. Destes, $24(77,4 \%)$ eram do sexo masculino; a média da idade foi de $68,6 \pm 9,8$ anos. Observou-se que 25 $(80,6 \%)$ indivíduos foram classificados como negros e 19 (61,3\%) eram casados, Tabela 1.

Dados sobre os valores pós broncodilatador (pós BD) e a gravidade da limitação de fluxo de ar, comorbidades e o perfil de uso de fármacos para a DPOC estão descritos na Tabela 2.

Nos quatro indivíduos com a classificação espirométrica GOLD 4 (VEF1 pós BD < 30\%) o TUG foi realizado em média em 9,3 2,5 segundos; nos 16 pacientes GOLD $3\left(30 \% \leq \mathrm{VEF}_{1}\right.$ pós $\left.\mathrm{BD}<50 \%\right)$ o tempo do TUG foi em média de 8,0+1,3 segundos; e nos 11 pacientes com a classificação espirométrica GOLD $2(50 \% \leq$ $V_{E F}$ pós $B D<80 \%$ ), o TUG foi realizado em $8,3 \pm 2,3$ segundos. Nenhum indivíduo da amostra foi classificado como GOLD 1. Não houve diferença estatística referente ao tempo do TUG entre os grupos GOLD encontrados na amostra (ANOVA, $p=0,5$ ).

\section{Reprodutibilidade do TUG}

Em relação à análise da reprodutibilidade intraobservador do TUG pelo $\mathrm{CCl}$, obteve-se $\alpha=0,897$ (IC 95\%: 0,$786 ; 0,950 ; p<0,0001$ ). A Figura 1 ilustra a dispersão de dados representando a reprodutibilidade intraobservador do desempenho, em segundos, no TUG 1 e TUG 2. As médias do tempo para desempenhar o TUG1 e TUG2 foram respectivamente de $8,3 \pm 1,8$ segundos e 7,9 $\pm 1,9$ segundos $(p=0,104)$. O valor da diferença entre as médias de tempo entre os testes TUG1 e TUG2 foi igual a 0,35 $\pm 1,15$ segundos, não sendo estatisticamente significante $(p=0,104)$. Três pacientes $(9,7 \%)$ tiveram um desempenho superior ao tempo de 11,2 segundos no TUG1, e uma $(3,2 \%)$ no TUG2.

A variabilidade no desempenho (tempo) de realização dos dois testes TUG está demonstrada na figura 2, pela disposição gráfica de Bland \& Altman. Dos 31 pacientes avaliados, $10(32,3 \%)$ desempenharam tempo no TUG1 = TUG2; $15(48,4 \%)$ desempenharam tempo TUG1 > TUG2 (reduziu o tempo na 2a prática, ou seja, houve melhora do desempenho); seis (19,3\%) desempenharam tempo TUG2 > TUG1 (aumento do tempo na $2^{\mathrm{a}}$ prática). Nenhum paciente necessitou de dispositivo de auxílio na marcha. 
Tabela 1. Características sociodemográficas dos pacientes com Doença Pulmonar Obstrutiva Crônica ( $n=31)$

\begin{tabular}{|c|c|c|}
\hline Características & Média \pm DP & $n(\%)$ \\
\hline Idade (anos) & $68,6 \pm 9,8$ & \\
\hline \multicolumn{3}{|l|}{ Sexo } \\
\hline Masculino & & $24(77,4)$ \\
\hline Feminino & & $7(22,6)$ \\
\hline \multicolumn{3}{|l|}{ Estado civil } \\
\hline Casado & & $19(61,3)$ \\
\hline Solteiro & & $8(25,8)$ \\
\hline Viúvo & & $4(12,9)$ \\
\hline \multicolumn{3}{|l|}{ Cor da pele } \\
\hline Branca & & $5(16,1)$ \\
\hline Negra & & $25(80,6)$ \\
\hline Indígena & & $1(3,2)$ \\
\hline \multicolumn{3}{|l|}{ IMC } \\
\hline Baixo peso & & $1(3,2)$ \\
\hline Eutrófico & & $12(38,7)$ \\
\hline Sobrepeso & & $14(45,2)$ \\
\hline Obeso & & $4(12,9)$ \\
\hline
\end{tabular}

Tabela 2. Características clínicas dos pacientes com Doença Pulmonar Obstrutiva Crônica, n=31

\begin{tabular}{|c|c|c|}
\hline Variável & Média (\%) & $\mathrm{DP}( \pm)$ \\
\hline \multicolumn{3}{|l|}{ Função Pulmonar } \\
\hline CVF (\% previsto pós BD) & 65,1 & 16,6 \\
\hline $\mathrm{VEF}_{1}(\%$ previsto pós $\mathrm{BD})$ & 45,9 & 14,9 \\
\hline $\mathrm{VEF}_{1} / \mathrm{CVF}$ & 59,0 & 10,8 \\
\hline \multirow[t]{2}{*}{$\mathrm{SpO}_{2}$} & 95,9 & 2,1 \\
\hline & n (31) & $\%$ \\
\hline \multicolumn{3}{|c|}{ Graus de limitação ao fluxo de ar (GOLD 2021) } \\
\hline \multicolumn{3}{|c|}{ Moderado } \\
\hline Grave & 11 & 35,8 \\
\hline \multirow{2}{*}{ Muito grave } & 16 & 51,6 \\
\hline & 4 & 12,6 \\
\hline Hipertensão arterial & 20 & 64,5 \\
\hline Diabetes mellitus & 4 & 12,9 \\
\hline \multicolumn{3}{|l|}{ Estado Tabágico } \\
\hline Nunca fumou & 3 & 9,7 \\
\hline Tabagista & 7 & 22,6 \\
\hline Ex- tabagista & 21 & 67,7 \\
\hline Uso de broncodilatador & 25 & 80,6 \\
\hline Uso de corticoide inalatório & 7 & 22,6 \\
\hline
\end{tabular}


Figura 1. Reprodutibilidade intraobservador do desempenho, em segundos, no TUG 1 e TUG $2(a=0,897 ;$ IC $95 \%$ : $0,786-0,950)$; $p<0,0001$. $n=31$

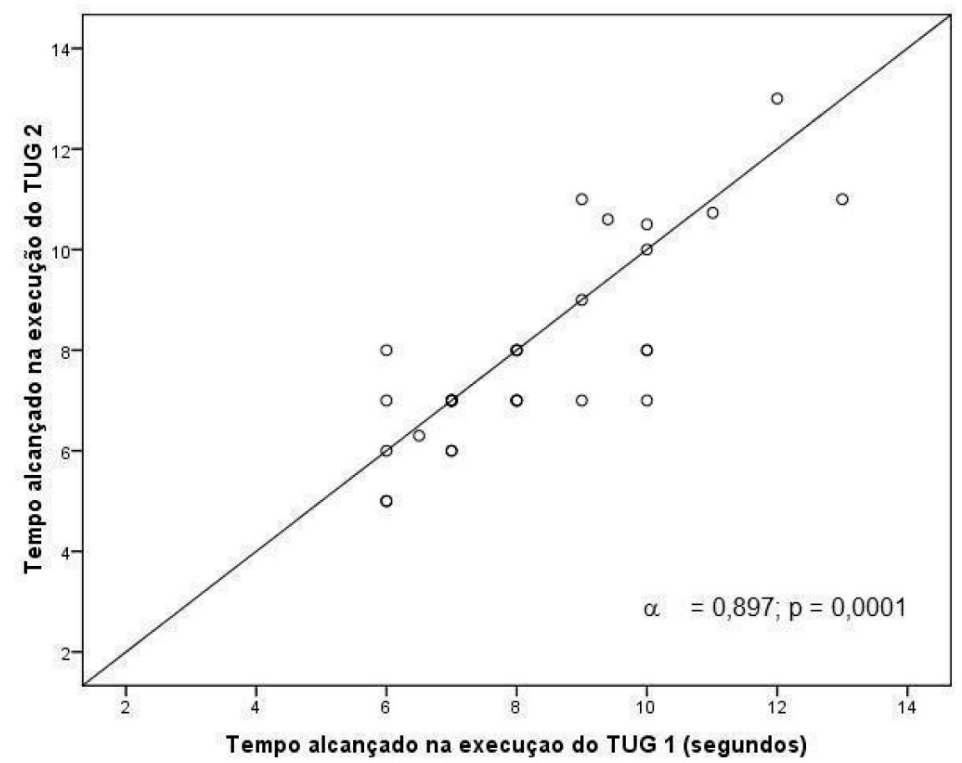

Figura 2. Disposição gráfica de Bland-Altman, da diferença entre os tempos dispendidos nas duas práticas do TUG (1 e 2$)$ em relação às médias individuais do desempenho (tempo) nos dois testes [(média: 0,35 segundos; IC 95\% (- 1,92; 2,61)]

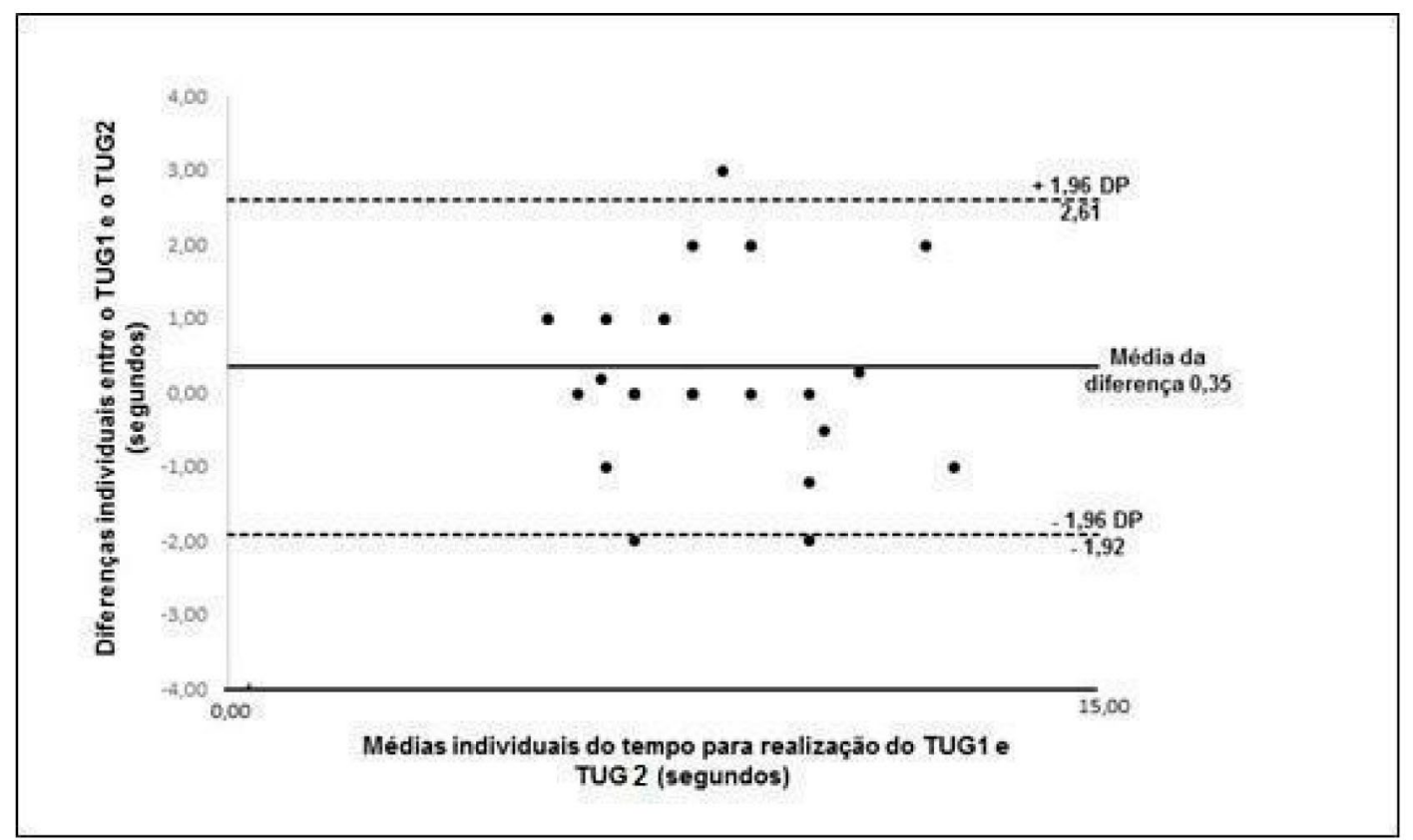




\section{Discussão}

O TUG é um teste amplamente utilizado em diversas populações devido à sua capacidade de identificar pessoas com risco de quedas e sua facilidade de aplicação, necessitando de poucos instrumentos para sua realização.,6,15 No presente estudo, verificou-se que a realização do TUG foi considerada de baixa complexidade e de excelente reprodutibilidade intraobservador. Foi também notada pequena variabilidade do teste em pacientes com DPOC, corroborando com dados de uma revisão sobre testes funcionais publicada recentemente, na qual foi indicado que a média da diferença entre os testes variou de 0,06 a 0,96 segundos., ${ }^{4,11,16}$ O TUG foi considerado de boa reprodutibilidade intraobservador neste estudo e em outras publicações da literatura, não somente para a DPOC, mas também na Insuficiência Cardíaca Crônica (ICC), Insuficiência Renal Crônica (IRC) e Acidente Vascular Cerebral (AVC). ${ }^{10,15}$

$\mathrm{Na}$ análise da variabilidade, verificou-se baixo valor de diferença média entre as duas mensurações do TUG, achado também encontrado em pessoas com doenças crônicas em estado avançado, incluindo a DPOC. $\frac{15}{}$ A aplicação da repetição do TUG por outro observador (reprodutibilidade inter observador) pode, contudo, resultar em variabilidade fora dos limites de concordância, como pode ser avaliado na literatura. $\stackrel{11}{ }$ Desta maneira, pode-se ser sugerido que em uma situação de repetição ou reavaliação pelo TUG (após uma intervenção, por exemplo), que se recomende que este teste seja executado pelo mesmo profissional de saúde.

O principal desfecho de medida do TUG é a mensuração do tempo, em segundos, e no presente estudo, este foi aferido por um cronômetro manual, sendo obtida uma boa reprodutibilidade intraobservador. Entretanto, sabe-se que o tempo cronometrado pode ser influenciado pela habilidade do avaliador. ${ }^{17} \mathrm{Na}$ literatura, pode-se encontrar estudos que sugerem a utilidade do uso de cronômetros automáticos como aspecto que melhore a confiabilidade do teste, aumentando o CCl. 17 Utilizando um cronômetro automático em um estudo, foi encontrado um $\mathrm{CCl}>0,88$, sendo o $\mathrm{CCl}>0,86$ usando um manual. ${ }^{17} \mathrm{Na}$ casuística deste estudo, o uso do cronômetro manual não parece ter alterado a reprodutibilidade do teste, bem como este aspecto aumenta a praticidade e reduz o custo de recursos para optar pelo TUG para avaliação da funcionalidade.

O número de repetições do TUG pode variar até três vezes na literatura, sendo identificada em um estudo, diferença estatística da primeira com a terceira avaliação, sugerindo um efeito aprendizado $\frac{15}{}$, algo não verificado na presente avaliação. Portanto, recomenda-se que o TUG seja executado por duas vezes, escolhendo-se o melhor desempenho e não havendo necessidade de uma terceira execução.11,15

É esperada uma redução do tempo no desempenho do TUG após suas repetições, como o encontrado em alguns estudos.4 Porém, fatores como os efeitos causados pela senilidade, baixa tolerância ao exercício físico na DPOC, assim como a diminuição de força do quadríceps podem desempenhar papel importante para a redução do desempenho em testes funcionais. ${ }^{3,18}$ Neste estudo, a maioria dos avaliados apresentou redução do tempo de realização no segundo teste, porém parte significante da amostra desempenhou o TUG1 = TUG2, e 19,3\% obtivereram TUG2 > TUG1. Estes achados podem apresentar relação direta com a baixa tolerância ao esforço, redução de força da musculatura dos membros inferiores, alterações de mobilidade e equilíbrio, como citado anteriormente, impactando no aumento do tempo de realização do TUG após repetições. ${ }^{3,18}$

Evidências científicas apontam para a redução da mobilidade funcional e função global ${ }^{19,20}$ causadas pela fadiga, disfunção muscular e redução da tolerância ao exercício, que podem comprometer também o equilíbrio, especialmente com a doença avançada. ${ }^{8}$ Em estados de exacerbação da DPOC, os indivíduos apresentam pior mobilidade avaliada pelo TUG em comparação a estado de não exacerbação. ${ }^{8,21}$ Neste trabalho, o resultado do teste nos indivíduos avaliados demonstrou-se abaixo do ponto de corte de 11,2 segundos ${ }^{15}$ preconizado para a população com DPOC, com exceção de um indivíduo na segunda prática, corroborando com um estudo que avaliou o equilíbrio em pessoas com a mesma condição de saúde $^{22}$, ou seja, a população estudada não apresentou riscos de queda ou déficit de equilíbrio, mesmo estando em sua maioria entre os estágios moderado a grave da doença, o que pode estar relacionado ao tamanho amostral reduzido. Este achado também pode ser justificado devido ao fato de que o estudo 
utilizado como referência ${ }^{14}$ para o ponto de corte no teste utilizou em sua amostra apenas pacientes em estado avançado da doença, e no presente trabalho não foi utilizado como critério de exclusão pacientes em estágio moderado. Além disso, cita-se a dificuldade de pessoas em estágios mais avançados da doença se deslocarem até os serviços de saúde.

Na amostra estudada, os níveis de limitação do fluxo aéreo proposto pela Iniciativa GOLD ${ }^{1}$ não identificaram diferenças estatisticamente no desempenho do teste TUG pelos indivíduos, reforçando a hipótese defendida por Pitta et al. ${ }^{2}$ e Cavalheri et al. ${ }^{23}$, que avaliaram a relação entre a obstrução aérea e o desempenho em atividades, mesmo quando verificados em graus mais avançados da DPOC. Deste modo, o desempenho no teste está mais intimamente relacionado com o comprometimento musculoesquelético já citado.,222

Tratou-se de um estudo que submeteu os participantes a um risco mínimo; e a avaliação foi conduzida com recursos simples e acessível, e de baixo custo. Indica-se como limitação o acesso a pacientes em estágios mais avançados da doença, e pela amostra ser de conveniência.

\section{Conclusão}

Após análise dos resultados, é possível inferir que o teste TUG possui excelente reprodutibilidade intraobservador e uma pequena variabilidade quando aplicadas as duas práticas a indivíduos com DPOC sendo a aplicação considerada factível para avaliação da mobilidade na prática do cuidado em saúde.

\section{Agradecimentos}

À Universidade do Estado da Bahia (UNEB), ao Conselho Nacional de Desenvolvimento Científico e Tecnológico (CNPq) e à Fundação de Amparo à Pesquisa do Estado da Bahia (FAPESB) pela oportunidade e concessão das bolsas para o Programa Institucional de Iniciação Científica (IC) / PROAF aos autores Henrique da Conceição Costa, Bárbara Silva dos Santos, Taís Santana Barbosa e Aline Gonçalves Miranda. À Fundação Maria Emília pela concessão de bolsa de apoio à produção científica ao autor Aquiles Assunção Camelier.

\section{Contribuições dos autores}

Costa HC e Santos BS participaram da concepção, delineamento, busca e análise estatística dos dados da pesquisa, interpretação dos resultados, redação do artigo científico. Santos NC participou da concepção, delineamento e busca de dados e revisão final. Barbosa TS e Miranda AG participaram da análise dos dados, interpretação dos resultados, redação do artigo científico. Camelier AA e Camelier FWR participaram da concepção, delineamento, busca e análise estatística dos dados da pesquisa, interpretação dos resultados, redação e revisão do artigo científico.

\section{Conflitos de interesses}

Nenhum conflito financeiro, legal ou político envolvendo terceiros (governo, empresas e fundações privadas, etc.) foi declarado para nenhum aspecto do trabalho submetido (incluindo, mas não se limitando a subvenções e financiamentos, participação em conselho consultivo, desenho de estudo, preparação de manuscrito, análise estatística, etc.). Camelier AC tem contrato de tempo parcial com a empresa GSK Brasil e recebe bolsa de apoio à Produção Científica da Fundação Maria Emília (desde março de 2020).

\section{Referências}

1. Global Initiative for Chronic Obstructive Lung Disease. Global Strategy for the Diagnosis, Management and Prevention of Chronic Obstructive Pulmonary Disease [Internet]. GOLD; 2021 [citado em 2021 jul. 20]. Disponível em: https://goldcopd. org/2021-gold-reports/

2. Pitta F, Troosters T, Probst VS, Langer D, Decramer M, Gosselink R. Are Patients With COPD More Active After Pulmonary Rehabilitation? Chest. 2008;134(2):273-80. https://doi.org/10.1378/ chest.07-2655

3. Kofod LM, Døssing M, Steentoft J, Kristensen MT. Resistance Training With Ankle Weight Cuffs Is Feasible in Patients With Acute Exacerbation of COPD. J Cardiopulm Rehabil Prev. 2017;37(1):4956. https://doi.org/10.1097/hcr.0000000000000230

4. Mesquita R, Wilke S, Smid DE, Janssen D, Franssen F, Probst VS. Measurement properties of the Timed Up \& Go test in patients with COPD. Chron Respir Dis. 2016;13(4)344-52. https://doi. org/10.1177/1479972316647178

5. Meriem M, Cherif J, Toujani S, Ouahchi Y, Hmida AB, Beji M. Sit-to-stand test and 6-min walking test correlation in patients with chronic obstructive pulmonary disease. Ann Thorac Med. 2015;10(4):269-73. https://doi.org/10.4103/1817-1737.165289

6. Bui K, Nyberg A, Maltais F, Saey D. Functional Tests in Chronic Obstructive Pulmonary Disease, Part 2: Measurement Properties. Seminar for Clinicians. Ann Am Thorac Soc. 2017 May;14(5):78594. https://doi.org/10.1513/annalsats.201609-734as 
7. Martinez BP, Gomes IB, Oliveira CS, Ramos IR, Rocha MD, Forgiarini Junior LA, et al. Accuracy of the Timed Up and Go test for predicting sarcopenia in elderly hospitalized patients. Clinics. 2015;70(5):369-72. https://doi.org/10.6061/clinics/2015(05)11

8. Crişan AF, Oancea C, Timar B, Fira-Mladinescu O, Tudorache V. Balance impairment in patients with COPD. PLoS One. 2015;10(3):e0120573. https://dx.doi.org/10.1371\%2Fjournal. pone. 0120573

9. Holland AE, Malaguti C, Hoffman M, Lahham A, Burge AT, Dowman L, et al. Home-based or remote exercise testing in chronic respiratory disease, during the COVID-19 pandemic and beyond: A rapid review. Chron Respir Dis. 2020;17:1479973120952418. https://doi. org/10.1177/1479973120952418

10. Hafsteinsdóttir TB, Rensink M, Schuurmans M. Clinimetric properties of the Timed Up and Go Test for patients with stroke: a systematic review. Top Stroke Rehabil. 2014;21(3):197-210. https://doi.org/10.1310/tsr2103-197

11. Marques A, Cruz J, Quina S, Regêncio M, Jácome C. Reliability, Agreement and Minimal Detectable Change of the Timed Up \& Go and the 10-Meter Walk Tests in Older Patients with COPD. COPD. 2016;13(3):279-87. https://doi.org/10.3109/15412555.2015.1079 $\underline{816}$

12. Mkacher W, Tabka Z, Trabelsi Y. Minimal Detectable Change for Balance Measurements in Patients With COPD. J Cardiopulm Rehabil Prev. 2017;37(3):223-8. https://doi.org/10.1097/ hcr. 0000000000000240

13. Portney LE, Watkins MP. Foundations of Clinical Research Applications to practice. 3a Ed. Nova Jersey: Preason Prentice Hall; 2009. p 87.

14. Fleiss JL. The Design and Analysis of Clinical Experiments [Internet]. Nova Jersey: John Wiley \& Sons, Inc.; 1986.

Disponível em: https://onlinelibrary.wiley.com/doi/ book/10.1002/9781118032923

15. Mesquita R, Janssen DJ, Wouters EF, Schols JM, Pitta F, Spruit MA. Within-day test-retest reliability of the Timed Up \& Go test in patients with advanced chronic organ failure. Arch Phys Med Rehabil. 2013;94(11):2131-8. https://doi.org/10.1016/j. apmr.2013.03.024
16. Al Haddad MA, John M, Hussain S, Bolton CE. Role of the Timed Up and Go Test in Patients With Chronic Obstructive Pulmonary Disease. J Cardiopulm Rehabil Prev. 2016;36(1):49-55. https://doi. org/10.1097/hcr.0000000000000143

17. Mateo CD, Madeira P, Muñoz FJD, Villafaina S, Carus PT, Parraca JA. The Automatic Assessment of Strength and Mobility in Older Adults: A Test-Retest Reliability Study. Medicina (Kaunas). 2019;55(6):270. https://doi.org/10.3390/medicina55060270

18. Butcher SJ, Pikaluk BJ, Chura RL, Walkner MJ, Farthing JP, Marciniuk DD. Associations between isokinetic muscle strength, high-level functional performance, and physiological parameters in patients with chronic obstructive pulmonary disease. Int J Chron Obstruct Pulmon Dis. 2012;(7):537-42. https://doi. org/10.2147/copd.s34170

19. Al Haddad MA, John M, Hussain S, Bolton CE. Role of the Timed Up and Go Test in Patients With Chronic Obstructive Pulmonary Disease. J Cardiopulm Rehabil Prev. 2016;36(1):49-55. https://doi. org/10.1097/hcr.0000000000000143

20. Cruz J, Marques A, Jácome C, Gabriel R, Figueiredo D. Global Functioning of COPD Patients With and Without Functional Balance Impairment: An Exploratory Analysis Based on the ICF Framework. COPD. 2015;12(2):207-16. https://doi.org/10.3109/154 $\underline{12555.2014 .933793}$

21. Tudorache E, Oancea C, Avram C, Fira-Mladinescu O, Petrescu L, Timar B. Balance impairment and systemic inflammation in chronic obstructive pulmonary disease. Int J Chron Obstruct Pulmon Dis. 2015;10:1847-52. https://doi.org/10.2147/copd. $\underline{\mathrm{s} 89814}$

22. Marques A, Jácome C, Cruz J, Gabriel R, Figueiredo D. Effects of a pulmonary rehabilitation program with balance training on patients with COPD. J Cardiopulm Rehabil Prev. 2015;35(2):154-8. https://doi.org/10.1097/hcr.0000000000000097

23. Cavalheri V, Hill K, Donaria L, Camillo CA, Pitta F. Maximum voluntary ventilation is more strongly associated with energy expenditure during simple activities of daily living than measures of airflow obstruction or respiratory muscle strength in patients with COPD. Chron Respir Dis. 2012;9(4):239-40. https://doi. org/10.1177/1479972312458681 